\title{
Comparison of $\gamma$ production data from thermal neutron capture on gadolinium with the Monte Carlo simulation
}

\section{Kaito HAGIWARA*}

Department of Physics, Okayama University, Okayama 700-8530, Japan

E-mail: K.hagiwarads.okayama-u.ac.jp

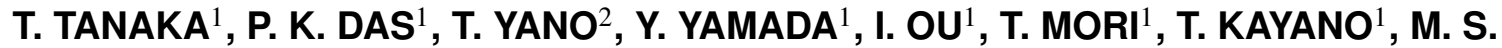
REEN $^{1}$, R. DHIR ${ }^{1}$, Y. KOSHIO ${ }^{1}$, M. SAKUDA ${ }^{1}$, A. KIMURA $^{3}$, S. NAKAMURA ${ }^{3}$, N. IWAMOTO $^{3}$ and H. HARADA ${ }^{3}$

${ }^{1}$ Department of Physics, Okayama University, Okayama 700-8530, Japan

${ }^{2}$ Department of Physics, Kobe University, Kobe Hyogo 657-8501, Japan

${ }^{3}$ Japan Atomic Energy Agency, 2-4 Shirakata Shirane, Tokai, Naka, Ibaraki 319-1195, Japan

The $\gamma$-ray spectrum produced from the thermal neutron capture on enriched gadolinium targets $\left({ }^{155} \mathrm{Gd}\right.$ and $\left.{ }^{157} \mathrm{Gd}\right)$ were measured using the ANNRI Germanium Spectrometer at J-PARC. We have also built a $\gamma$-ray emission model of ${ }^{158} \mathrm{Gd}$ decay, in which $\gamma$ rays were classified into discrete prompt $\gamma$ rays and continuum spectrum. We compared the data and our model, and found a fair agreement between them at the level of $26 \%$ over the $\gamma$-ray energy from 0.2 to $8.0 \mathrm{MeV}$. In this proceedings, the measured spectra and the model are described.

The 3rd International Symposium on" Quest for the Origin of Particles and the Universe"

5-7 January 2017

Nagoya University, Japan

\footnotetext{
* Speaker.
} 


\section{Introduction}

Gadolinium-157 ( $\left.{ }^{157} \mathrm{Gd}\right)$ has the largest thermal neutron capture cross section among all stable nuclei and Gadolinium-155 ( $\left.{ }^{155} \mathrm{Gd}\right)$ also has large cross section since they have a resonance in the neutron capture in the thermal neutron energy region $\left(26.8 \mathrm{meV}\right.$ for ${ }^{155} \mathrm{Gd}$ and $31.4 \mathrm{meV}$ for $\left.{ }^{157} \mathrm{Gd}\right)$ [四]. The neutron capture reaction $\operatorname{Gd}(\mathrm{n}, \gamma)$ is expected to produce about four $\gamma$ rays, which have the total energy of about $8 \mathrm{MeV}$. These $\mathrm{Gd}(\mathrm{n}, \gamma)$ reactions are described as

$$
\begin{aligned}
& \mathrm{n}+{ }^{155} \mathrm{Gd} \rightarrow{ }^{156} \mathrm{Gd}^{*} \rightarrow{ }^{156} \mathrm{Gd}+\gamma \text { rays } \quad(8.5 \mathrm{MeV} \text { in total), } \\
& \mathrm{n}+{ }^{157} \mathrm{Gd} \rightarrow{ }^{158} \mathrm{Gd}^{*} \rightarrow{ }^{158} \mathrm{Gd}+\gamma \text { rays } \quad(7.9 \mathrm{MeV} \text { in total). }
\end{aligned}
$$

A new project (SK-Gd) for anti-electron-neutrino detection using Gd-loaded water Cherenkov detector was proposed for the Super-Kamiokande collaboration [《], 园]. In this project, the inverse beta decay reaction $\left(\bar{v}_{e}+\mathrm{p} \rightarrow \mathrm{e}^{+}+\mathrm{n}\right)$ can be identified by the coincidence of a prompt positron signal and a delayed $\gamma$-ray signal from the neutron capture on $\mathrm{Gd}$. The present experiment was performed at the J-PARC in order to measure the $\gamma$ rays from the $\mathrm{Gd}(\mathrm{n}, \gamma)$ reaction at high precision.

\section{Experiment}

The experiment (2014B0124) was conducted in December, 2014, using the ANNRI detector [䧃]. The ANNRI detector is located at the Beam Line No.4 (BL04) in the MLF [可]. The BL04 provides a neutron beam from $1 \mathrm{meV}$ to $10 \mathrm{eV}$. The ANNRI detector is composed of two Ge clusters and BGO counters. A Ge cluster consists of seven hexagonal Ge crystals, each of which measures the energy and the arrival time of $\gamma$ rays. The BGO counters surround the Ge clusters, which are used as anti-coincidence shields. The details of the detector can be found elsewhere [ 6 ].

We used two kinds of enriched gadolinium oxide powder, ${ }^{155} \mathrm{Gd}_{2} \mathrm{O}_{3}$ and ${ }^{157} \mathrm{Gd}_{2} \mathrm{O}_{3}$ (Table $\mathbb{~}$ ), attached to a target holder. The ANNRI detector was calibrated using $\gamma$ rays from four radioactive sources $\left({ }^{22} \mathrm{Na},{ }^{60} \mathrm{Co},{ }^{137} \mathrm{Cs}\right.$, and $\left.{ }^{152} \mathrm{Eu}\right)$ and prompt $\gamma$ rays from ${ }^{35} \mathrm{Cl}(\mathrm{n}, \gamma)$ reaction. We select events only when at least one Ge crystal has pulse height greater than $100 \mathrm{keV}$ and no surrounding BGO counters have pulse height greater than $100 \mathrm{keV}$. Table $\square$ shows the summary of the data sets.

Table 1: Isotopic composition (\%) of the Gd oxide powder used in the experiment.

\begin{tabular}{|c|c|c|c|c|c|c|}
\hline Target & ${ }^{154} \mathrm{Gd}$ & ${ }^{155} \mathrm{Gd}$ & ${ }^{156} \mathrm{Gd}$ & ${ }^{157} \mathrm{Gd}$ & ${ }^{158} \mathrm{Gd}$ & ${ }^{160} \mathrm{Gd}$ \\
\hline \hline${ }^{155} \mathrm{Gd}_{2} \mathrm{O}_{3}$ & 0.5 & 91.85 & 5.87 & 0.81 & 0.65 & 0.27 \\
\hline${ }^{157} \mathrm{Gd}_{2} \mathrm{O}_{3}$ & 0.05 & 0.30 & 1.63 & 88.4 & 9.02 & 0.60 \\
\hline
\end{tabular}

Table 2: Data sets taken in the experiment.

\begin{tabular}{|c|c|c||c|c|c|}
\hline Target & Time & Number of Events & Source & Time & Number of Events \\
\hline \hline${ }^{155} \mathrm{Gd}_{2} \mathrm{O}_{3}$ & 38 hours & $3.1 \times 10^{9}$ & ${ }^{22} \mathrm{Na}$ & 5 minutes & $1.5 \times 10^{7}$ \\
${ }^{157} \mathrm{Gd}_{2} \mathrm{O}_{3}$ & 55 hours & $4.6 \times 10^{9}$ & ${ }^{60} \mathrm{Co}$ & 18 hours & $8.8 \times 10^{7}$ \\
$\mathrm{NaCl}$ & 4 hours & $1.3 \times 10^{8}$ & ${ }^{137} \mathrm{Cs}$ & 30 minutes & $2.1 \times 10^{6}$ \\
Empty & 6 hours & $1.3 \times 10^{7}$ & ${ }^{152} \mathrm{Eu}$ & 7 hours & $2.3 \times 10^{7}$ \\
\hline
\end{tabular}


We first show the energy spectrum of single $\gamma$-ray events in Figure $\mathbb{W}$. Single $\gamma$-ray events are those in which only one Ge crystal is hit in an event. The fraction of single $\gamma$-ray events out of the total events is $77 \%$.

\section{Modeling $\gamma$-ray Emission}

We used two Geant4-Based Monte Carlo (MC) simulations for $\gamma$-ray emission from $\operatorname{Gd}(\mathrm{n}, \gamma)$ reaction. One of them is the standard option of Geant4 [Q, [8]. The other is a GLG4sim model [Q] which is developed for KamLAND [10]].

We have built our model following the GLG4sim scheme, where the $\gamma$ rays were classified into two parts: discrete prompt $\gamma$ rays and continuum spectrum. For the prompt $\gamma$ rays, their photo-peak energies in the range from 5 to $8 \mathrm{MeV}$ and the relative intensities were estimated from our data. We then select events where two Ge crystals are hit. Out of those events, we tag one prompt $\gamma$ ray and look at the second $\gamma$ ray (secondary $\gamma$ ray). The secondary $\gamma$ rays and their intensities are also estimated and are summarized in Table [3]. The relative intensity (\%) is normalized by total number of single $\gamma$ rays. We have found that their intensities are consistent with the already published values within the errors [W]. The sum of intensities of discrete $\gamma$ rays is $2.9 \%$.

Table 3: Discrete $\gamma$-ray energies (MeV) and their relative intensities (\%), normalized by the total number of single $\gamma$-rays observed in our data.

\begin{tabular}{|c|c|c|c|c|c|}
\hline \multicolumn{5}{|c|}{ Discrete $\gamma$ rays $(\mathrm{MeV})$} & \multirow{2}{*}{ Relative Intensity (\%) } \\
\hline Prompt $\gamma$ ray & \multicolumn{3}{|c|}{ Secondary $\gamma$ ray } & $0.0023 \pm 0.0002$ \\
\hline 7.937 & - & - & - & - & $0.0097 \pm 0.0006$ \\
\hline 7.857 & 0.080 & - & - & - & $0.0085 \pm 0.0005$ \\
\hline 6.960 & - & - & - & - & $0.053 \pm 03$ \\
\hline 6.914 & 0.944 & 0.080 & - & - & $0.51 \pm 0.03$ \\
\hline \multirow{2}{*}{6.750} & 1.187 & - & - & - & $0.51 \pm 0.03$ \\
\cline { 2 - 6 } & 1.107 & 0.080 & - & - & $0.068 \pm 0.007$ \\
\hline \multirow{2}{*}{6.672} & 1.187 & 0.080 & - & - & $0.012 \pm 0.002$ \\
\cline { 2 - 6 } & 1.004 & 0.182 & 0.080 & - & $0.052 \pm 0.006$ \\
\hline \multirow{3}{*}{6.420} & 1.517 & - & - & - & $0.058 \pm 0.006$ \\
\cline { 2 - 6 } & 1.438 & 0.080 & - & - & $0.027 \pm 0.004$ \\
\cline { 2 - 6 } & 1.256 & 0.182 & 0.080 & - & $0.066 \pm 0.003$ \\
\hline 6.001 & - & - & - & - & $0.20 \pm 0.01$ \\
\hline \multirow{3}{*}{5.903} & 1.010 & 0.944 & 0.080 & - & $0.12 \pm 0.01$ \\
\cline { 2 - 6 } & 0.875 & 0.898 & 0.182 & 0.080 & $0.11 \pm 0.01$ \\
\cline { 2 - 6 } & 0.769 & 1.186 & 0.080 & - & $0.064 \pm 0.006$ \\
\cline { 2 - 6 } & 0.676 & 1.097 & 0.182 & 0.080 & $0.083 \pm 0.003$ \\
\hline 5.784 & - & - & - & - & $0.26 \pm 0.01$ \\
\hline 5.669 & - & - & - & - & $0.099 \pm 0.004$ \\
\hline 5.595 & - & - & - & - & $0.068 \pm 0.003$ \\
\hline 5.543 & - & - & - & - & $0.251 \pm 0.009$ \\
\hline 5.436 & - & - & - & - & \\
\hline 5.167 & - & - & - & - & \\
\hline
\end{tabular}


The continuum spectrum can be described in terms of the nuclear level density (NLD) and a set of photon strength functions (PSF). The transition probability $P\left(E_{a}, E_{b}\right)$ from the initial energy level $E_{a}$ to the final energy level $E_{b}$, emitting the $\gamma$-ray energy $E_{\gamma}\left(=E_{a}-E_{b}\right)$, can be written using Fermi Golden Rule as

$$
\begin{aligned}
P\left(E_{a}, E_{b}\right) \Delta E_{b} & =\frac{T\left(E_{\gamma}\right) \rho\left(E_{b}\right) \Delta E_{b}}{\int_{0}^{E_{a}} T\left(E_{\gamma}\right) \rho\left(E_{b}\right) \mathrm{d} E_{b}}, \text { with } \\
T\left(E_{\gamma}\right) & =2 \pi E_{\gamma}^{3} f\left(E_{\gamma}, T\right),
\end{aligned}
$$

where $T\left(E_{\gamma}\right), f\left(E_{\gamma}, T\right), \rho\left(E_{b}\right)$ and $T$ are the gamma-ray transmission coefficient, PSF, NLD, and the nuclear temperature, respectively. Electromagnetic decay from the neutron resonances is dominated by dipole transitions. The PSFs for deformed nuclei like Gd are described by the sum of two Lorentzian terms [[2]

$$
f\left(E_{\gamma}, T\right)=\sum_{i=1}^{2}\left[\frac{E_{\gamma} \Gamma_{i}\left(E_{\gamma}, T\right)}{\left(E_{\gamma}^{2}-E_{i}^{2}\right)^{2}+E_{\gamma}^{2} \Gamma_{i}^{2}\left(E_{\gamma}, T=0\right)}+0.7 \frac{\Gamma_{i}\left(E_{\gamma}=0, T\right)}{E_{i}^{3}}\right] \sigma_{i} \Gamma_{i},
$$

where $E_{i}, \Gamma_{i}\left(E_{\gamma}, T\right)$, and $\sigma_{i}$ are the parameters of the giant electric dipole resonances which have two components $(i=1,2)$. Here, the $\gamma$-ray- and temperature-dependent width is given by

$$
\Gamma_{i}\left(E_{\gamma}, T\right)=\left[k_{0}+\frac{E_{\gamma}-\varepsilon_{0}^{\gamma}}{E_{i}-\varepsilon_{0}^{\gamma}}\left(1-k_{0}\right)\right] \frac{\Gamma_{i}}{E_{i}^{2}}\left(E_{\gamma}^{2}+4 \pi^{2} T^{2}\right),
$$

and guarantees $\Gamma_{i}(E, 0)=\Gamma_{i}$. We used the parameters from the database of RIPL-2 [ए[3] ; $E_{1,2}=$ 11.7 and $14.9 \mathrm{MeV}, \Gamma_{1,2}=2.6$ and $3.8 \mathrm{MeV}, \sigma_{1,2}=165$ and $249 \mathrm{mb}, \varepsilon_{0}^{\gamma}=4.5 \mathrm{MeV}$, and $k_{0}=4.0$. The HFB model was also used for NLD in the database of RIPL-3 [14]].

We compared the $\gamma$ rays generated by the model with our data, as shown in Figure $\square$. We obtained the fraction of the continuum to be $97.1 \%$ and that of the discrete $\gamma$ rays to be $2.9 \%$. The $\gamma$ rays below $5 \mathrm{MeV}$ energy region is dominated by the continuum spectrum. We also note that the contribution (red color) of prompt $\gamma$ rays (including their compton scattering) to the total spectrum is very small, except for the energy region above $5 \mathrm{MeV}$, as shown in Figure $\mathrm{W}$.

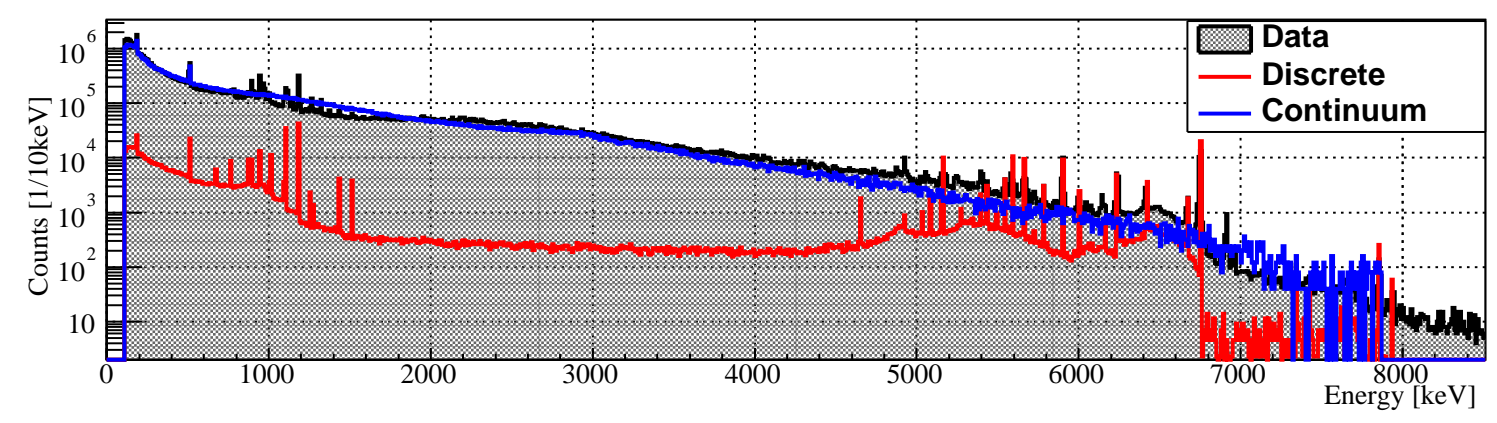

Figure 1: Energy spectrum of single $\gamma$ rays from ${ }^{157} \mathrm{Gd}(\mathrm{n}, \gamma)$ reaction. Shaded histogram stands for data, while colored histograms (red and blue) stand for discrete $\gamma$ rays and continuum spectrum predicted by our MC model, respectively. 


\section{Result and Summary}

The $\gamma$-ray emission model is implemented into our Geant 4 detector simulation. Figure \shows the comparison between data and three models (Geant4 standard option, GLG4sim model, and our model). Our model agrees with the data within $26 \%$, when data and MC are compared at every $0.5 \mathrm{MeV}$ from $1 \mathrm{MeV}$ to $9 \mathrm{MeV}$. Compared to GLG4sim model, the agreement of the data and our model in the energy region between 0.2 to $8.0 \mathrm{MeV}$ was improved by a factor of three.

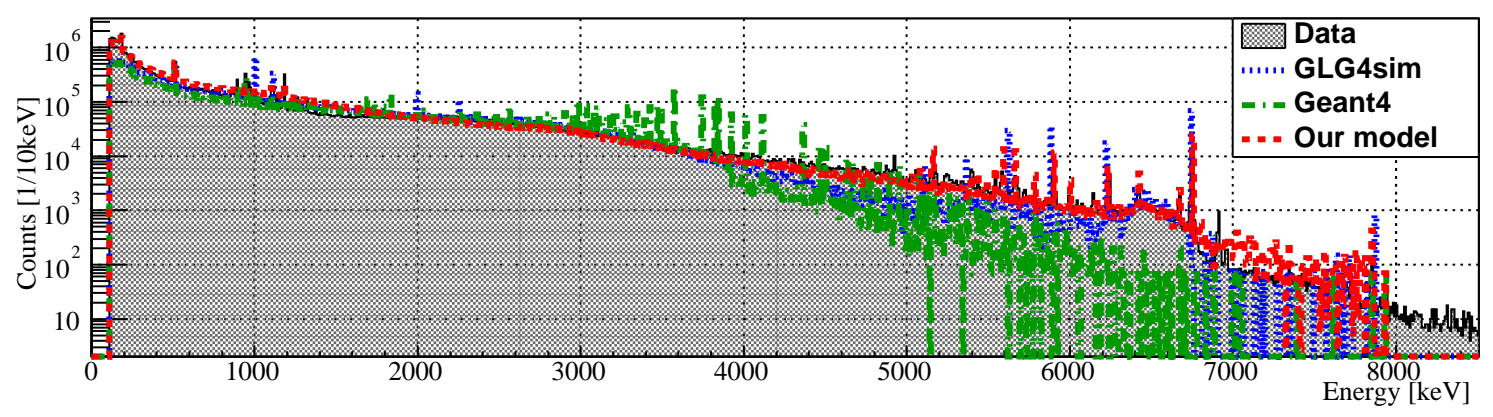

Figure 2: Energy spectrum of single $\gamma$ rays from ${ }^{157} \mathrm{Gd}(\mathrm{n}, \gamma)$ reaction. Shaded histogram stands for our data (same as those given in Figure 1), while colored histograms stand for predictions by GLG4sim (dotted blue), Geant4 standard option (dashed green), and our model (dashed red).

The ${ }^{155} \mathrm{Gd}(\mathrm{n}, \gamma)$ data are also being analyzed [U]]. Both ${ }^{155} \mathrm{Gd}$ and ${ }^{157} \mathrm{Gd}$ data will be published elsewhere soon. This work is supported by JSPS Grant-in-Aid for Scientific Research on Innovative Areas (Research in a proposed research area) No. 26104006.

\section{References}

[1] S. F. Mughabghab: Atlas of Neutron Resonances (Elsevier, Amsterdam, 2006).

[2] J. F. Beacom and M. R. Vagins: Phys. Rev. Lett 93 (2004) 171101.

[3] H. Watanabe et al. (Super-K Collab.): Astropart. Phys. 31 (2009) 320.

[4] A. Kimura et al:: J. Nucl. Sci. Technol. 49 [7] (2012) 708.

[5] Y. K. M. Igasira and M. Oshima: Nucl. Instrum. Methods Phys. Res. A 600 (2009) 332.

[6] I.Ou et al. AIP Conference Proceedings 1594, 351 (2014); doi: 10.1063/1.4874094.

[7] S. Agostinelli et al.: Nucl. Instrum. Methods Phys. Res. A 506 (2003) 250.

[8] Geant4 Physics Reference Manual: <http://geant4.cern. ch/G4UsersDocuments/UsersGuides / PhysicsReferenceManual/html/PhysicsReferenceManual.html>.

[9] GLG4sim page: <http://neutrino.phys.ksu. edu/ GLG4sim/>.

[10] K. Eguchi et al.: Phys. Rev. Lett. 90 (2003) 021802.

[11] P. K. Das et al.: In this proceedings.

[12] J. Kopecky, M. Uhl and R. E. Chrien: Phys. Rev. C 47 (1993) 312.

[13] T. Belgya et al:: IAEA-TECDOC-1506.

[14] R. Capote et al.: Nucl. Data Sheets 110 (2009) 3107. 